\title{
Optimal periodic control of a drug delivery system
}

\author{
Subbarao Varigonda ${ }^{1}$, Tryphon T. Georgiou ${ }^{2}$, Ronald A. Siegel ${ }^{3}$, Prodromos Daoutidis ${ }^{4}$
}

\begin{abstract}
Administration of certain drugs at a steady rate results in deterioration of drug effect, also known as drug tolerance. Periodic delivery is an attractive option for minimizing tolerance and maximizing the desired effect of such drugs. In this paper, periodic drug infusion strategies for maximizing a time averaged measure of drug effect are investigated. A simple pharmacokinetic-pharmacodynamic (PKPD) model of a system exhibiting tolerance is considered and optimal periodic control theory is employed. First, regions of PKPD parameter space in which periodic infusion provides a locally improved average effect compared to steady infusion are characterized using the so-called $\pi$ test. Then, optimal drug delivery strategies, obtained using two different computational approaches, are presented for a representative set of parameter values, and insight is provided into the results. The first method, proposed by the authors, is based on the notion of differential flatness, while the second is based on the standard shooting method for dynamic optimization problems.
\end{abstract}

\section{Introduction}

The past several decades have seen many improvements in drug therapy. Not only have a new classes of drugs been discovered, developed, and commercialized, but fruitful attention has been placed on the spatiotemporal pattern of drug administration, or drug delivery. With proper design, dosage forms can be programmed to release drugs in ways that are rationally matched to the pharmacokinetics, pharmacodynamics, and toxicodynamics of the drug.

For most drugs, it is reasonable to assume that, at steady state, drug effect is a function of present drug concentration in the blood. For such drugs constant rate, or zero-order delivery, is considered optimal, because it leads to a constant blood concentration

\footnotetext{
1 Corresponding author. Currently with United Technologies Research Center, East Hartford, CT. Email: varigos@utrc.utc.com

2 Department of Electrical and Computer Engineering, University of Minnesota

3 Departments of Pharmaceutics and Biomedical Engineering, University of Minnesota

4 Department of Chemical Engineering and Materials Science, University of Minnesota
}

that is sufficiently high to guarantee drug efficacy, but low enough to avoid toxic side effects. Large efforts have been therefore been made to develop zero-order controlled release dosage forms such as tablets and capsules.

Exceptions to the zero-order rule exist, however. For certain drugs, effect depends not only on the present concentration, but also on the previous history of exposure. When steady exposure leads to attenuated drug effect, the patient is said to develop tolerance to the drug. While tolerance is best known to be associated with addiction and drug abuse, it must also be considered in more mundane clinical settings. As a classic example, nitroglycerin skin patches were developed in the 1980s to provide zeroorder prophyllaxis against angina. These patches were intended to be worn for 24 hours and replaced daily, providing zero order release throughout. It was found, however, that protection diminished after about $12 \mathrm{hr}$, due to development of tolerance. As a result, patients are now advised to wear the patches for $1 / 2$ day, followed by a $1 / 2$ day without the patch [18]. In another example, gonadotropin releasing hormone $(\mathrm{GnRH})$ deficiency, which leads to 
reproductive disorders in both males and females, must be treated with periodic pulses (period 1-2 $\mathrm{hr}$ ), as steady infusion leads to nearly complete suppression of gonadal function [24]. This suppression can be regarded as rapid development of tolerance to the hormone.

While the need for periodic drug delivery in the presence of drug tolerance is established in practice, theoretical considerations of periodic dosing to optimize average effect over time are relatively rare $[10,17,1,28]$. In this paper, a simple pharmacokinetic/pharmacodynamic (PKPD) model of a drug is considered and the question whether periodic drug infusion is more effective in a time averaged sense is investigated. In section 2 the problem is formulated as an optimal periodic control (OPC) problem so that powerful analytical and computational techniques developed for OPC problems can be employed $[4,2,14,27]$. In section 3 , the so-called $\pi$-test is used to assess whether small periodic variations around the optimal steady state (OSS) can improve performance, a situation known as local properness. The region in a two dimensional parameter space where the problem is locally proper is computed.

Since the $\pi$-test is local and only concerns small sinusoidal inputs, more elaborate computations using dynamic optimization methods are introduced in section 4 to obtain a solution to the OPC problem. Since state constraints are periodic, with initially unknown period, the OPC problem is computationally more difficult than other dynamic optimization problems. The solution to the OPC problem is obtained using two computational methods, both of which produce a consistent solution. The first method is based on flatness as described in [27] and the second is a shooting method implemented within a commercially available process modeling software tool, gPROMS ${ }^{\mathrm{TM}}[22]$.

\section{Drug delivery system model and formulation of the OPC problem}

Several models of drug tolerance have appeared in recent decades, using mathematical representations of receptor/effector down regulation [23,10], delayed indirect inhibition $[11,8]$ and physiological counterregulation $[20,1]$. Here, a variation of a model for tolerance to the cardio-accelerating effect of nicotine [21] is considered. In this model, tolerance is attributed to the buildup of a drug metabolite that acts as antagonist to the drug, thus reducing drug effect over time. As will be seen, periodic drug delivery leads to better average performance than steady delivery, given this model.

The system consists of two linked one compartment models, one for drug and one for metabolite, with nonnegative drug input and so-called massless conversion of drug to metabolite [21]. Drug effect is represented by a saturable, Langmuir binding function with noncompetitive antagonism by the metabolite. All variables, including concentrations, drug effect, and time are appropriately nondimensionalized. The (dimensionless) drug and metabolite (antagonist) concentrations are denoted, respectively, by $c$ and $a$.

The nondimensionalized dynamics are linear and given by

$\dot{c}=-c+u$

$\dot{a}=k_{a}(c-a)$

where $u$ is the drug infusion rate and $k_{a}$ is the rate constant for antagonist elimination. Drug effect is described by

$E(c, a)=\frac{c}{(1+c)\left(1+a / a^{*}\right)}$

where $a^{*}$ is a measure of the relative potency of antagonist compared to drug. The variables $c, a$ and $u$ are all constrained to be positive. In addition there is an upper bound on $u$ reflecting the maximal rate at which the drug can be delivered.

As an initial consideration, the steady state of the system resulting from a constant infusion of drug, for any given $u(t)=\bar{u}$ is given by $\bar{c}=\bar{a}=\bar{u}$ and $\bar{E}=\frac{\bar{u}}{(1+\bar{u})\left(1+\bar{u} / a^{*}\right)}$. Steady state effect is maximized when $\bar{u}=\sqrt{a^{*}}$, and hence $\max (\bar{E})=\frac{\sqrt{a^{*}}}{\left(1+\sqrt{a^{*}}\right)^{2}}$.

A simple objective would be to target $E$ within a prescribed interval $\left[E_{1}, E_{2}\right]$, which can be interpreted for the nicotine system as the desired interval for the heart rate. Obviously, when $\max (\bar{E})<E_{1}$, stationary input will ultimately lead to ineffective drug action due to tolerance. (The case $\max (\bar{E})>$ $E_{2}$ is trivial in the present model as it is possible to reduce $\bar{E}$ at will.) This binary objective may be too restrictive, however, and anyway it is unsuitable for the techniques used in the present work. In its place, we define a smoothed, fuzzy indicator function

$I(E)=\frac{\left(E / E_{1}\right)^{\gamma}}{\left[1+\left(E / E_{1}\right)^{\gamma}\right]\left[1+\left(E / E_{2}\right)^{2 \gamma}\right]}$.

which is close to unity when $E \in\left[E_{1}, E_{2}\right]$ and close to zero otherwise. The parameter $\gamma$ determines 


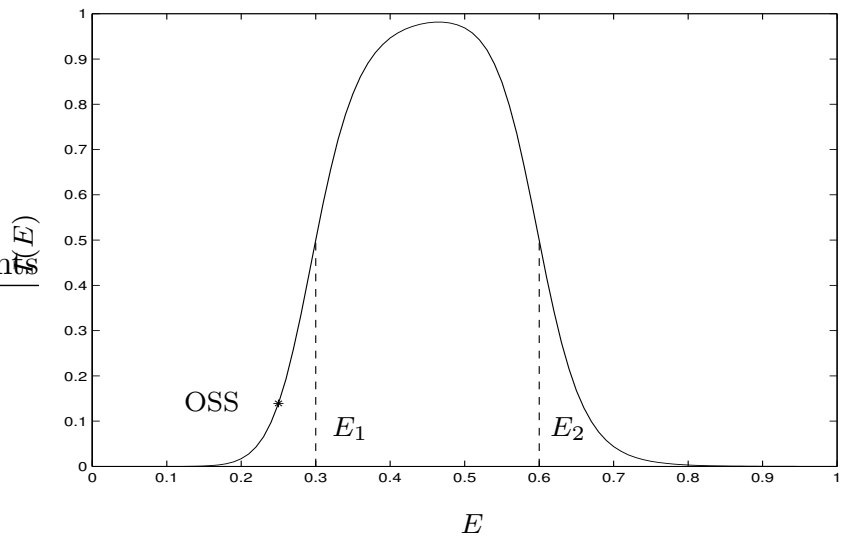

Fig. 1. Plot of the indicator function $I(E)$ for $E_{1}=0.3$, $E_{2}=0.6$, and $\gamma=10$. The desired range of the effect $E$, $\left[E_{1}, E_{2}\right]$ and the OSS value for $K_{a}=0.1$ and $a^{*}=1$ are marked.

the sharpness of the transition of the indicator function near $E_{1}$ and $E_{2}$. The indicator function tends to the binary objective when $\gamma \rightarrow \infty$. A plot of $I(E)$ is shown in Fig 1 , with $E_{1}=0.3, E_{2}=0.6$, and $\gamma=10$.

In the present context, a more realistic objective is to maximize the time average of the drug effect indicator, $I$ with respect to the drug infusion rate, $u$. Both steady and periodic profiles for $u$ are considered. When infusion is steady, this average will be $I(\bar{E}(\bar{u}, \bar{u}))$, which will take the maximal value $I(\max (\bar{E}))$ provided that one is restricted to the increasing part of the $I$ function. This will be called the optimal steady state (OSS) solution, or $J_{O S S}$. When $u$ is periodic, the time average of the indicator over one period is

$J=\frac{1}{T} \int_{0}^{T} I(E(c(t), a(t))) d t$.

The period $T$ is unknown and needs to be determined from optimization. The internal states of the system, $c$ and $a$, are required to be periodic as well, with period $T$. This results in an optimal periodic control (OPC) problem, and the resulting maximal value of the objective function under periodic input will be called $J_{O P C}$

\section{Analysis using the $\pi$ test}

When a periodically varying $u$ provides a better average drug effect than the OSS solution, the OPC problem is said to be proper. If performance improvements can be obtained by small sinusoidal pertur- bations of the input around the OSS, then the OPC problem is said to be locally proper. Local properness implies properness but the converse is need not be true. The so-called $\pi$-test can be used to determine local properness of an OPC problem [3,2].

Consider the OPC problem of minimizing $J=$ $g(y)$ over the inputs, $u(\cdot)$ and the period, $T$ for $\mathrm{dy}-$ namical system described by $\dot{x}=f(x, u)$ with averaged outputs $y=\frac{1}{T} \int_{0}^{T} \phi(x, u) d t$. For the present problem, $x=[c, a]^{\prime}$, where prime indicates transpose. Define the Hamiltonian of the system as

$H(x, u, y, \lambda, \mu)=g(y)+\lambda^{\prime} f(x, u)+\mu^{\prime}(\phi(x, u)-y)$

where $\lambda(\cdot), \mu$ are the Lagrange multipliers. (The sign of $\mu$ is different from that used in [2]. This definition ensures consistency of the OSS and OPC Hamiltonians). Let $G(s)$ be the transfer function corresponding to the linearized dynamics at the OSS i.e., $G(s)=\left(s I-\bar{f}_{x}\right)^{-1} \bar{f}_{u}$ where overbar denote the OSS value. Then the $\pi$-test for the local properness of OSS requires that the self-adjoint matrix $\pi(\omega)$ defined by

$$
\begin{aligned}
\pi(\omega)= & G^{\prime}(-j \omega) \bar{H}_{x x} G(j \omega)+\bar{H}_{u x} G(j \omega) \\
& +G^{\prime}(-j \omega) \bar{H}_{x u}+\bar{H}_{u u}
\end{aligned}
$$

be partially negative for frequency, $\omega>0$ [2]. Since the present system is single input-single output, $\pi$ is actually a scalar and the criterion reduces to $\pi(\omega)<$ 0 for some $\omega>0$. The $\pi$-test determines whether small sinusoidal perturbations of the input $u$ around the OSS value $\bar{u}$ improve performance.

As an example, consider the nominal parameter values: $k_{a}=0.1, a^{*}=1, E_{1}=0.3, E_{2}=0.6$ and $\gamma=$ 10. Also assume the following bounds on infusion rate: $u_{\min }=0<u_{\max }=10$. In this case the OSS is at $c=a=u=1, \max (\bar{E})=0.25$, and the OSS objective function is $I(0.25)=0.139$. Note that, for the model considered here, $\bar{H}_{x u}=\bar{H}_{u x}^{\prime}=0$ and $\bar{H}_{u u}=$ 0 and $\pi(\omega)$ simplifies to $G^{\prime}(-j \omega) \bar{H}_{x x} G(j \omega)$. Since $G(s)$ is a strictly proper transfer function, $\pi(\omega) \rightarrow 0$ as $\omega \rightarrow 0$. A plot of $\pi(\omega)$, shown in Fig 2, displays a range of frequency where $\pi(\omega)$ takes on negative values, indicating that the system is proper, and periodic operation can improve the time averaged objective function reflecting drug effect.

A study of the influence of the parameter values $k_{a}$ and $a^{*}$, which characterize the rapidity of metabolite accumulation and potency, respectively, on the prediction of the $\pi$-test reveals that the region over which the system is locally proper (i.e., with small amplitude periodic drug delivery being superior to 


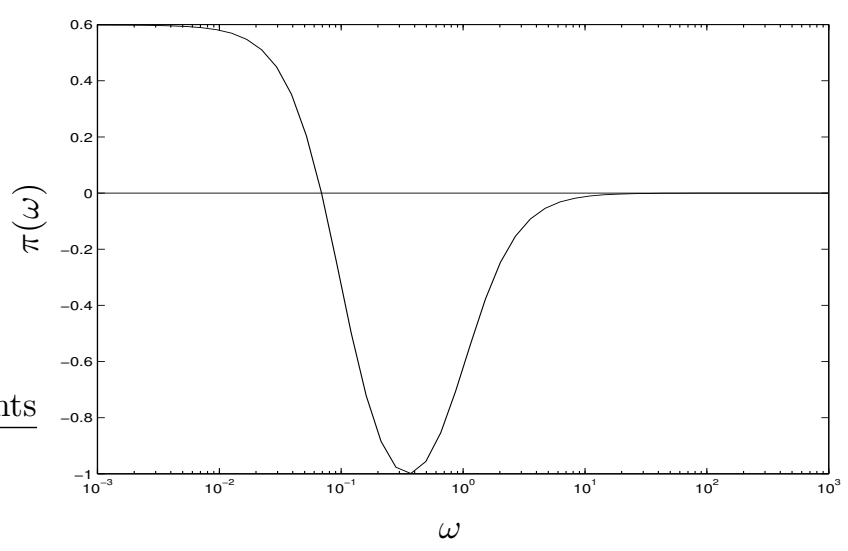

Fig. 2. Result of the $\pi$ test for the drug effect model with $k_{a}=0.1$ and $a^{*}=1$

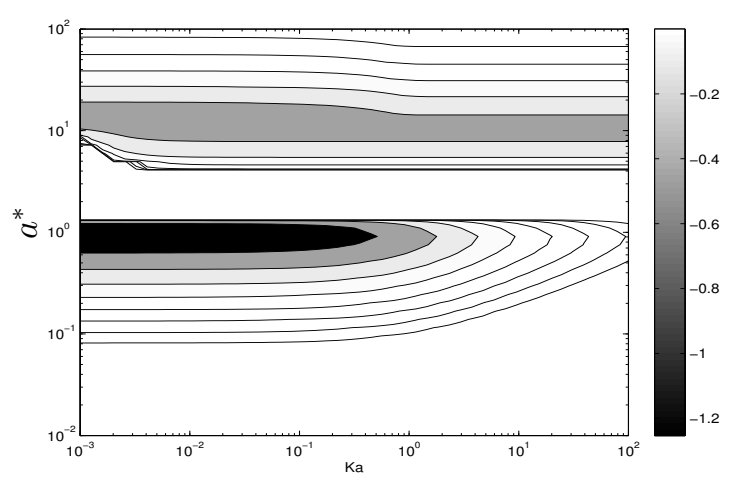

Fig. 3. Contour plot of $\min _{\omega} \pi(\omega)$ in the $k_{a}-a^{*}$ space (log-log scale). The darker regions indicate greater improvement in objective from small sinusoidal forcing relative to steady state

steady delivery) is rather extensive. This is not surprising since for the model considered, $\min _{w} \pi(\omega) \leq$ 0 always holds and local properness is violated only when the equality holds. Fig 3 shows a contour plot of $\min _{w} \pi(\omega)$ in the $k_{a}-a^{*}$ space. The darker regions in the figure represent regions of greatest improvement from small sinusoidal forcing.

The surface plot of $\min _{\omega} \pi(\omega)$ in Fig 4 shows the extent of improvement that can be obtained with small amplitude periodic inputs in the $k_{a}-a^{*}$ parameter space. Although local properness exists in most of the space, there is more significant improvement in areas with the lowest $\min _{w} \pi(\omega)$.

\section{Computation of the optimal periodic drug delivery strategy}

Since the $\pi$-test is local, it cannot be used to determine the exact shape of the periodic control signal

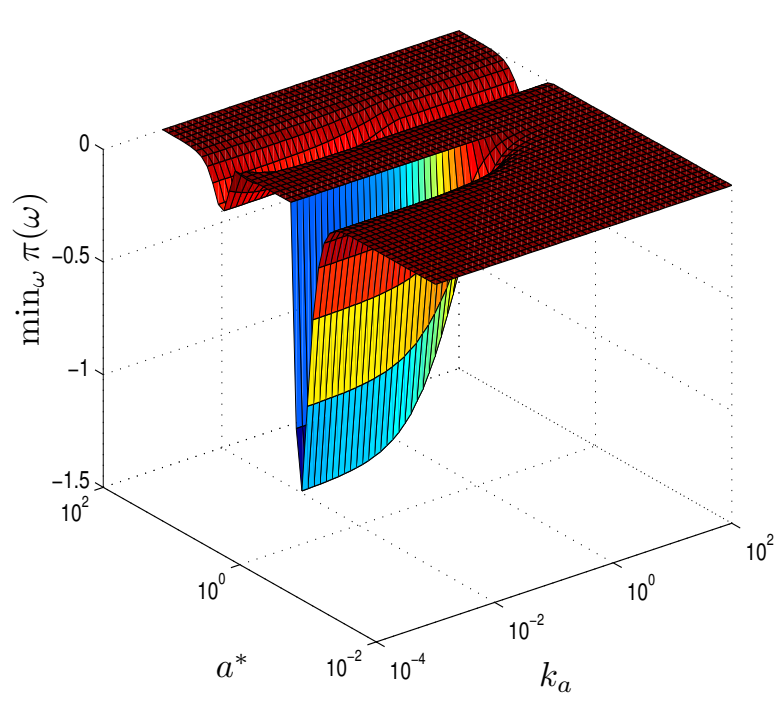

Fig. 4. Surface plot of $\min _{\omega} \pi(\omega)$ as a function of $k_{a}$ and $a^{*}$. Large negative values of $\min \pi$ indicate stronger gains from periodic delivery.

that maximizes the time averaged objection function reflecting drug effect. Computational methods for dynamic optimization, with some modifications, need to be employed for this purpose [5,14]. Computation for the OPC problem is, in general, much more difficult than for a regular optimal control problem, since the state constraints on the state are periodic and the time period is also unknown.

We compute and compare the solution to the OPC problem formulated in 2 using two different methods. The intent is to understand the features of the optimal solution qualitatively, ensure that the solution is sufficiently robust to variations in the particular computational approach one chooses and provide a physical justification for the optimal drug delivery profile. A comparison of the numerical and/or computational performance of different algorithms for optimal control or optimal periodic control problems is outside the scope of this paper.

\subsection{Solution using flatness based method}

First, we employ a method based on differential flatness described in [27] to obtain the optimal periodic drug infusion strategy. Differential flatness (or simply, flatness), is a property of a dynamical system that is related to the concepts of absolute equivalence and dynamic feedback linearizability $[26,13]$. The system described by $\dot{x}=f(x, u)$ is differentially 
flat if there exist outputs $\xi \in R^{m}$ depending on $x, u$ and a finite number of time derivatives of $u$ (i.e., $\left.\xi=h\left(x, u, \dot{u}, \ldots, u^{(\rho)}\right)\right)$ such that $x, u$ can be expressed solely as functions of $\xi$ and its derivatives up to order $\kappa[7]$. The vector $\xi$ must be of the same dimension as $u$ and is called the flat output of the system. An optimal control problem on a flat system can be reformulated as a static optimization problem for efficient computation $[9,16,12]$. The need for integration of differential equations is removed by restating the problem in terms of the so-called flat outputs. A computational method for OPC problems using flatness has been presented in [27], and this approach is used here for the current drug delivery problem to compute the OPC solution.

The dynamics in (1)-(2) are linear and flat with output $\xi=a$. The states and input are determined from $\Xi:=[\xi, \dot{\xi}, \ddot{\xi}]$ using the relations

$$
\begin{aligned}
& a=\xi \\
& c=\frac{\dot{\xi}}{K_{a}}+\xi \\
& u=\frac{\ddot{\xi}}{K_{a}}+\left(1+\frac{1}{K_{a}}\right) \dot{\xi}+\xi .
\end{aligned}
$$

The highest order derivative of the flat output is parametrized using $2 N+1$ Fourier basis functions:

$$
\begin{aligned}
\xi^{(\kappa)}(t, \alpha)= & \alpha_{1}+\sum_{i=1}^{N}\left[\alpha_{2 i} \sin (i \omega t)\right. \\
& \left.+\alpha_{2 i+1} \cos (i \omega t)\right]
\end{aligned}
$$

where $\omega=2 \pi / T$ and $\alpha=\left[\alpha_{1}, \alpha_{2}, \ldots, \alpha_{N}\right]^{\prime}$. Then, lower order derivatives $\xi^{(\kappa-1)}, \ldots, \xi$ are obtained by successively integrating Eq. 4 (e.g. using quadrature or Simpson's rule), $\kappa$ times, with integration constant $\beta_{j}$ introduced during the $j^{\text {th }}$ integration. Imposing periodicity constraints on the states, one obtains $\alpha_{1}=0$ and $\beta_{j}=0$ for all $j=1, \ldots, \kappa-1$ since these coefficients lead to polynomial terms in $\xi(t)$. Thus there are only $2 N+2$ unknown parameters, namely, $T, \alpha_{2}, \ldots, \alpha_{2 N+1}$ and $\beta_{\kappa}$ to be determined by optimization. For convenience, these parameters are gathered into the vector

$\theta:=\left[T, \alpha_{2}, \ldots, \alpha_{2 N+1}, \beta_{\kappa}\right]^{\prime}$.

Several local optima were found for the system and the best solution obtained after several runs with different initial guesses is taken as the globally optimal solution. This solution, obtained using the flatness algorithm, with $N=20$ Fourier harmonics is shown in Fig 5 on the left side. System parameters were set to their nominal values $\left(k_{a}=1\right.$, $\left.a^{*}=10, E_{1}=0.3, E_{2}=0.6, \gamma=10\right)$. It should be noted that other locally optimal solutions exist and may be reached depending on the initial guess for $\theta$. The initial guess used and the final solution obtained for the global optimum parameter vector $\theta$ were $\theta_{0}=[18,0.01,0.03,0, \ldots, 0.0 .76]^{\prime}$ and $\theta=$ $[17.8138,0.0051,0.0295,-0.0058, \ldots, 0.0023,0.744]^{\prime}$ respectively. The average of the indicator under periodic operation $\left(J_{O P C}\right)$ is approximately $0.3537 \mathrm{in}$ contrast with the value, $J_{O S S}=0.1390$ for steady state operation. The improvement is obviously due to the dwell time of the optimal periodic solution in the high effect region.

\subsection{Solution using control parametrization and shooting method}

We now employ a very different computational method for the OPC problem that uses a discrete representation of the control signal, integration of the system dynamic equations to perform the optimization of cost subject to state periodicity and other constraints. We model the problem in a commercial off-the shelf modeling software, gPROMS $^{\mathrm{TM}}[22]$ and utilize the dynamic optimization algorithm provided within this tool. Two solvers based on control vector parametrization (CVP) are provided in gPROMS ${ }^{\mathrm{TM}}$ for dynamic optimization. These are CVP_SS (single shooting) and CVP_MS (multiple shooting). CVP stands for control vector parametrization and refers to the fact that the infinite dimensional control signals $u(t)$ are assumed to be of a certain shape (e.g., piecewise linear and piecewise constant) and are represented in terms of a finite number of parameters. The CVP_SS (single shooting) and CVP_MS (multiple shooting) algorithms implemented in gPROMS ${ }^{\mathrm{TM}}$ are described in the gPROMS ${ }^{\mathrm{TM}}$ Introductory and Advanced User Guides.

In order to solve the OPC problem using gPROMS $^{\mathrm{TM}}$, the problem was reformulated as a fixed time optimal control problem by rescaling time. Thus, the rescaled period was fixed at unity while the actual time period $T$ became an optimization parameter appearing on the right hand side of the differential equations. This rescaling enabled the objective function $J$ to be correctly evaluated. The solution obtained using the CVP_SS method with 5 piecewise linear segments for $u$ is shown in 

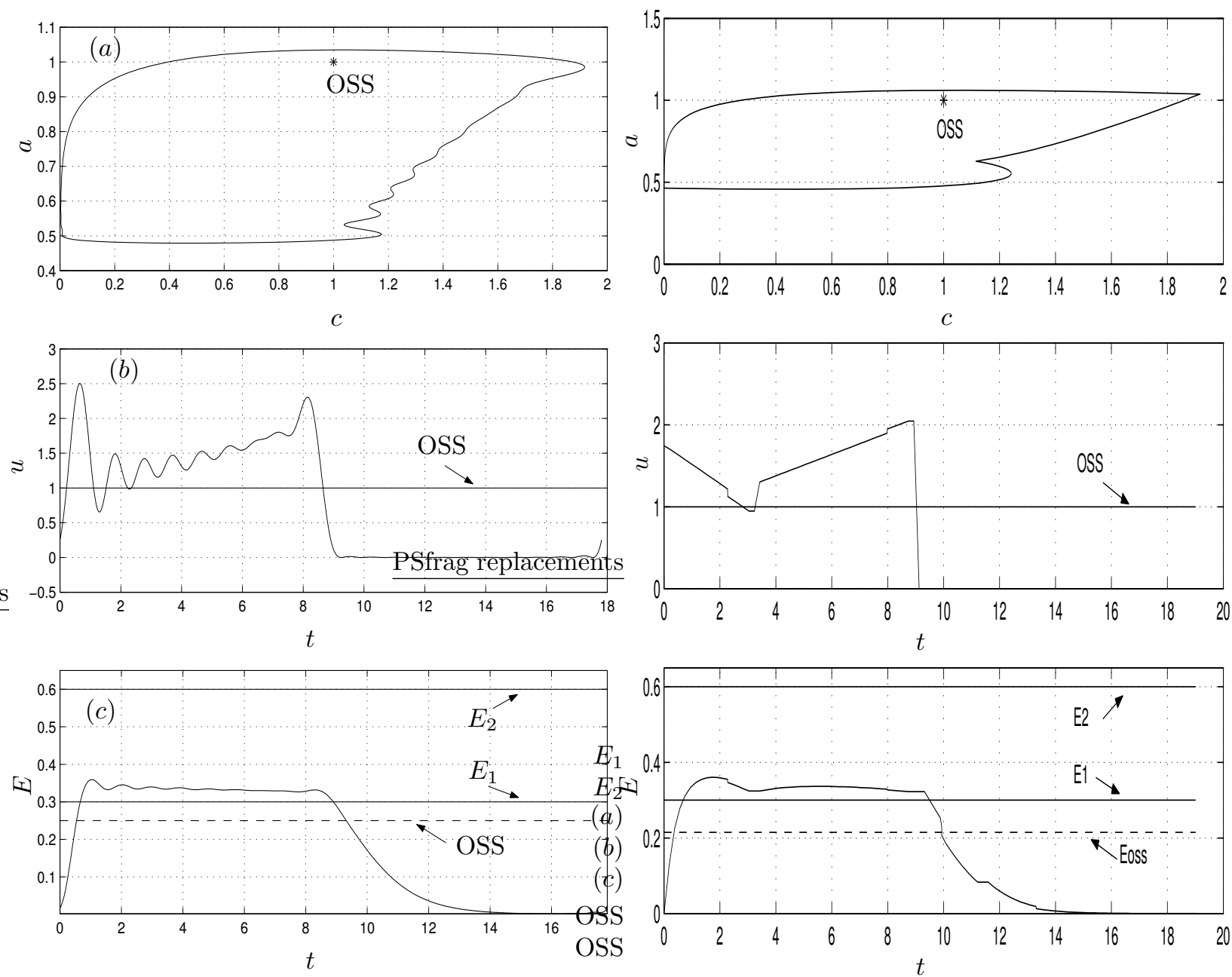

Fig. 5. Optimal periodic drug infusion strategy computed using the flatness method (left) and a shooting method in gPROMS ${ }^{\mathrm{TM}}$ (right): (a) state space, $(b)$ drug infusion rate and (c) drug effect

Fig 5 on the right. Once again, several local optima were found. The best solution found by the authors in several runs with different initial guesses had a cost of $J_{O P C}=0.3510$ and is reported here. The similarity in the shape of the trajectory in state space, the input profile and the shape of the drug effect between the two solutions in Fig 5 is evident. The differences seen near discontinuities are mainly due to the difference in the choice of basis functions (sum of smooth sinusoids vs. piecewise linear representation).

\section{Discussion}

The numerical improvement in the indicator $I(E)$ itself is not a significant factor since a higher magnitude of improvement can be obtained by using an indicator function that is steeper around the OSS. However, the qualitative features of the solution merit attention.

The solution is not of bang-bang type, but conforms to the intuitive idea that in the presence of tolerance, the best strategy is to first administer a "priming" dose, which brings drug effect into the desired range. Thereafter, drug should be administered at a rate just high enough to maintain effect in that range. Any higher infusion rate will ultimately accelerate the accumulation of the antagonizing metabolite. As metabolite eventually accumulates, drug must be infused at an accelerated rate to compensate. There will be a diminishing return, however, due to the saturability of response with respect to drug concentration. At some point, the best strategy is to stop infusion, and allow both drug and 
metabolite levels to decay to a point where the process can be started over again. This general pattern of drug release has actually been implemented to compensate for tolerance in an oral once-a-day controlled release formulation of methylphenidate $\mathrm{HCl}$, a drug used to treat attention deficit hyperactivity disorder [15]. While the system dynamics are somewhat more complicated than those presented in the present idealized system, we expect that the general features of the solutions calculated here will hold for a large class of systems involving drug tolerance.

While periodic drug delivery as an optimizing principle was discussed here in the context of drug tolerance, the periodic mode has been considered by others as a means for treating cancer. It has been argued that periodic delivery of cell cycle phasespecific chemotherapeutic agents, when matched to the turnover kinetics of cancer cells, will preferentially kill these cells, while reducing toxicity to normal cells, whose cycles are typically much slower $[6,25,19]$.

\section{Conclusion}

A simple pharmacokinetic, pharmacodynamic model of a drug that exhibits tolerance has been proposed and an optimal periodic control problem to determine a periodic drug infusion strategy that improves the drug effect is formulated. The local properness of the OPC problem was established using the $\pi$-test. The region in the parameter space where the problem is locally proper was also determined using the $\pi$-test. The optimal periodic drug infusion rate as a function of time was computed using two very different methods and a consistent solution was obtained which was intuitively explained. It was noted that periodic drug delivery has applications beyond the context of drug tolerance and these are opportunities for further studies using rigorous model based design techniques.

Periodic operation has the potential to provide significantly better performance over optimum steady state for some systems as is demonstrated for the drug delivery application here. In general, this is feasible when there are admissible regions of state space with favorable objective function "field" away from the steady state manifold and by suitable forcing of the dynamics of the system, these regions become accessible and enable a better time-average performance. The vast literature on the optimal periodic control reveals a significant gap between the mathematical theory and the understanding of the physical mechanisms and we hope this work will inspire further research in this direction.

\section{References}

[1] J. A. Bauer, J. P. Balthasar, H.-L. Fung, Application of pharmacodynamic modeling for designing time-variant dosing regiments to overcome nitroglycerin tolerance in experimental heart failure, Pharmaceut. Res. 14 (1997) 1140-1145.

[2] D. S. Bernstein, E. G. Gilbert, Optimal periodic control: The $\pi$ test revisited, IEEE Trans. Automat. Contr. AC25 (4) (1980) 673-684.

[3] S. Bittanti, G. Fronza, G. Guardabassi, Periodic control: A frequency domain approach, IEEE Trans. Automat. Contr. AC-18 (1) (1973) 33-38.

[4] S. Bittanti, G. Guardabassi, Optimal periodic control and periodic systems analysis: An overview, in: Proc. of the IEEE CDC, Athens, Greece, 1986.

[5] A. E. Bryson, Dynamic Optimization, Addison Wesley, 1999.

[6] B. F. Dibrov, A. M. Zhabotinsky, Y. A. Neyfakh, M. P. Orlova, L. I. Churikova, Mathematical model of cancer chemotherapy. periodic schedules of phasespecific cytotoxic-agent administration increasing the selectivity of therapy, Math. Biosc. 73 (1985) 1-31.

[7] M. Fliess, et al., Flatness and defect of nonlinear systems: introductory theory and examples, Int. J. Control 61 (6) (1995) 1327-1361.

[8] J. V. S. Gobburu, W. J. Jusko, Role of dosage regimen in controlling indirect pharmacodynamic responses, Adv. Drug Deliv. Revs. 33 (1998) 221-233.

[9] S. Kansal, J. F. Forbes, M. Guay, Constrained optimization of nonlinear, dynamic chemical processesa normalized form approach, in: Preprints of IFAC ADCHEM, vol. 2, Pisa, Italy, 2000.

[10] Y.-X. Li, A. Goldbeter, Frequency specificity in intercellular communication, Biophys. J. 55 (1) (1989) $125-145$.

URL http://www.pubmedcentral.nih.gov/ articlerender.fcgi?artid $=1330448$

[11] E. E. B. M., V. Licko, A model eliciting transient responses, Amer. J. Physiol. 246 (1984) R114-R121.

[12] R. Mahadevan, S. K. Agrawal, F. J. Doyle III, A flatness based approach to optimization in fed-batch bioreactors, in: Preprints of IFAC ADCHEM, vol. 1, Pisa, Italy, 2000.

[13] P. Martin, R. M. Murray, P. Rouchon, Flat systems, equivalence and trajectory generation, Tech. rep., CDS Technical report (2003).

URL http://www.cds.caltech.edu/ murray/papers/ 2003d_mmr03-cds.html

[14] H. Maurer, C. Büskens, G. Feichtinger, Solution techniques for periodic control problems: A case study in production planning, Optimal Control Appl. and Methods 19 (3) (1998) 185-203.

[15] N. B. Modi, B. Lindemulder, S. K. Gupta, Single- and multiple-dose pharmacokinetics of an oral once-a-day osmotic controlled-release OROSR (methylphenidate $\mathrm{HCl}$ ) formulation, J. Clin. Pharmacol. 40 (2000) 379388. 
[16] J. Oldenburg, W. Marquardt, Dynamic optimization based on higher order differential model representations, in: Preprints of IFAC ADCHEM, vol. 2, Pisa, Italy, 2000.

[17] K. Park, D. Verotta, S. K. Gupta, L. B. Sheiner, Use of a pharmacokinetic/pharmacodynamic model to design an optimal dose input profile, J. Pharmacokinet. Biopharm. 26 (1998) 471-492.

[18] J. O. Parker, M. H. Amies, R. W. Hawkinson, J. M. Heilman, A. J. Hougham, M. C. Vollmer, R. R. Wilson, Intermittent transdermal nitroglycerin therapy in angina pectoris. clinically effective without tolerance or rebound, Circulation 91 (1995) 1368-1374.

[19] R. S. Parker, F. J. I. Doyle, Control-relevant modeling in drug delivery, Adv. Drug Deliv. Revs. 48 (2001) 211228.

[20] A. Peper, C. A. Grimbergen, J. W. Kraal, J. H. Englebart, An approach to the modeling of the tolerance mechanism in the drug effect. I: The drug effect as a disturbance of regulations, J. Theor. Biol. 127 (1987) 413-426.

[21] H. C. Porchet, N. L. Benowitz, L. Sheiner, Pharmacodynamic model of tolerance: application to nicotine, J. Pharmacol. Exp. Therap. 244 (1988) 231236.

[22] Process Systems Enterprise (PSE), gPROMS: General process modeling system (2007).

URL http://www.psenterprise.com

[23] R. B. Raffa, R. Tallarida, The concept of a changing receptor concentration: Implications for the theory of drug action, J. Theor. Biol. 115 (1985) 625-632.

[24] N. Santoro, M. Filicori, W. F. J. Crowley, Hypogonadotropic disorders in men and women: Diagnosis and therapy with pulsatile gonadotropinreleasing hormone, Endocr. Revs. 7 (1985) 11-23.

[25] M. K. Sundareshan, R. S. Acharya, Optimal control in cancer therapy by simultaneous consideration of normal and cancer cell proliferation kinetics, Int. J. Systems Sci. 15 (1984) 773-776.

[26] M. van Nieuwstadt, M. Rathinam, R. M. Murray, Differential flatness and absolute equivalence of nonlinear control systems, SIAM J. Control Optim. 36 (4) (1998) 1225-1239.

[27] S. Varigonda, T. T. Georgiou, P. Daoutidis, Numerical solution of the optimal periodic control problem using differential flatness, IEEE Trans. Automat. Contr. 49 (2) (2004) 271-5.

[28] E. O. Wang, J. P. Balthasar, H.-L. Fung, Pharmacodynamics of in vivo nitroglycerin tolerance in normal conscious rats: Effects of dose and dosing protocol, Pharmaceut. Res. 21 (2004) 114-120. 